\section{Does inflammation predict radiographic progression in hand osteoarthritis?}

Dear Editor,

We read with deep interest the article by Mathiessen et al ${ }^{1}$ aimed to determine whether ultrasound predicts radiographic hand osteoarthritis (OA) progression after 5 years. This prospective study suggested that ultrasound-detected grey-scale synovitis and power Doppler signals were significantly associated with radiographic progression after 5 years. We really appreciate the work conducted by the authors and the findings support the use of ultrasound as a tool to detect patients with hand OA who are likely to progress. However, there are some worthwhile issues that need exploration.

We need to be very careful when assessing the risk factors of OA progression which easily leads to paradoxical phenomena. ${ }^{2}$ The study conducted by Mathiessen et $a l^{1}$ included 78 patients with hand $\mathrm{OA}$ at baseline, and found $17.9 \%$ joints experienced radiographic progression during the follow-up time. They further indicated that ultrasound-detected inflammation at baseline was significantly associated with radiographic progression of OA after 5 years. As we all know, inflammation has been considered as a risk factor for OA. ${ }^{3}$ However, synovitis and power Doppler were found in only $16.6 \%$ and $2.5 \%$ of the 78 included participants, respectively, which means only a fraction of the hand OA participants can at least partly be attributed to inflammation. In other words, only these small number of people were susceptible to inflammation. On further examining the effect of inflammation on OA progression, it is easy to speculate the positive findings if the inflammation still exists during the 5-year follow-up time but there are more people who are diagnosed with hand OA without ultrasound-detected inflammation at baseline. There definitely exist some other unmeasured OA risk factors for these patients. When examining the effect of ultrasound-detected inflammation on OA progression, it turned out to compare the effect of inflammation versus other unmeasured risk factors which may bias the results. If this speculation does exist, the number of 78 participants may be too small to reach a valid conclusion. Maybe it is better to examine the effect of new-onset inflammation on the progression of OA.

In addition to all the above, there are some other issues that need to be mentioned. First, we hypothesised that the inflammation continued to affect OA progression but the authors only reported the ultrasound-detected inflammation at baseline. We are not sure whether the inflammation has persisted during the 5 years of follow-up time. Second, the authors did not report the drug use of the included 78 participants, especially the use of anti-inflammation drugs. On the basis of the small number of participants, this may influence the results. Third, we have no idea whether there is a need to adjust for follow-up time. Further explanation about this adjustment is worthy of expectation.

We respect the great contributions of the authors and we would be very interested in the authors' response to these issues.

\section{Hui Li, Hua Liu}

Department of Orthopaedics, Xiangya Hospital, Central South University, Changsha, Hunan Province, China

Correspondence to Associate Professor Hua Liu, Department of Orthopaedics, Xiangya Hospital, Central South University, Xiangya Road, Changsha, Hunan Province 410008, China; 2941153178@qq.com

Contributors HLi: concept, writing; HLiu: concept, revising.

Competing interests None declared.

Provenance and peer review Not commissioned; internally peer reviewed.

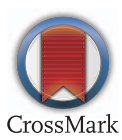

To cite Li H, Liu H. Ann Rheum Dis 2017;76:e20.

Received 29 November 2016

Accepted 1 December 2016

Published Online First 13 January 2017

Ann Rheum Dis 2017;76:e20. doi:10.1136/annrheumdis-2016-210893

\section{REFERENCES}

1 Mathiessen A, Slatkowsky-Christensen B, Kvien TK, et al. Ultrasound-detected inflammation predicts radiographic progression in hand osteoarthritis after 5 years. Ann Rheum Dis 2016;75:825-30.

2 Zhang $Y$, Neogi T, Hunter $D$, et al. What effect is really being measured? An alternative explanation of paradoxical phenomena in studies of osteoarthritis progression. Arthritis Care Res (Hoboken) 2014;66:658-61.

3 Robinson WH, Lepus CM, Wang Q, et al. Low-grade inflammation as a key mediator of the pathogenesis of osteoarthritis. Nat Rev Rheumatol 2016;12:580-92. 